\title{
Determinación de la presión venosa intra-acceso
}

\section{Raquel Muñoz Pérez - Anna Pérez Gil}

Centro de diálisis. DIAVERUM. Baix Llobregat. L' Hospitalet de Llobregat

\section{Resumen}

La medición de la presión venosa intraacceso es un método de vigilancia vascular que facilita la detección temprana de las disfunciones de las fístulas arteriovenosas. Nuestro objetivo fue describir como utilizamos este método de detección y exponer nuestra experiencia en el seguimiento de 31 pacientes.

En 9 pacientes se observó un aumento puntual de la presión venosa intraaceso, y a 4 se les realizó una angioplastia preventiva efectiva. En 7 pacientes que continuadamente presentaban un aumento de la presión intraaceso se trombosó el acceso vascular no siendo recuperable por angiorradiología. El aumento de la presión intraaceso puede facilitar la detección precoz de problemas en el acceso vascular.

\section{PALABRAS CLAVE:}

- ANGIOPLASTIA

PRESIÓN VENOSA

- HEMODIÁLISIS

- FÍSTULA ARTERIOVENOSA

- PRÓTESIS VASCULAR

\section{Determining intra-access venous pressure}

\section{Abstract}

Measurement of intra-access venous pressure is a vascular monitoring method that facilitates early

\section{Correspondencia: \\ Raquel Muñoz Pérez \\ DIAVERUM Catalunya C.D. Baix Llobregat \\ C/ Amadeo Torner 109 \\ 08901 L' Hospitalet de Llobregat (Barcelona) raquelmu81@hotmail.com}

detection of dysfunctions in arteriovenous fistulas. Our aim was to show how we use this detection method and describe our experience in monitoring 31 patients.

In 9 patients a one-off increase in intra-access venous pressure was observed, and in 4 patients an effective preventive angioplasty was carried out. In 7 patients who continually showed an increase in intra-access pressure, vascular access was thrombosed and not recoverable by angio radiology. The increase in intra-access pressure can facilitate the early detection of problems in vascular access.

\section{KEY WORDS:}

- ANGIOPLASTY

- VENOUS PRESSURE

HAEMODIALYSIS

ARTERIOVENOUS FISTULA

VASCULAR PROSTHESIS

\section{Introducción}

El funcionamiento adecuado del acceso vascular es fundamental para la práctica de la hemodiálisis por lo que su control es esencial ${ }^{1-3}$, la determinación de la presión venosa intraacceso (PVIA) es un método de vigilancia y seguimiento del acceso vascular con la intención de detectar la posible disfunción, en especial de las prótesis sintéticas y prevenir la trombosis de dichos accesos ${ }^{4}$.

En nuestro centro esta medida se realiza desde el año 2005. Surgió a partir de la detección de un aumento de trombosis de los accesos vasculares con prótesis sintética, sin causa evidente, por lo que se tomó la decisión de realizar la determinación de la PVIA mensualmente, con la intención de detectar precozmente las disfunciones de este tipo de ac- 
cesos vasculares y realizar angioplastias, evitando trombosis que requieren de la colocación de un catéter, una pérdida de calidad de la diálisis y mayor gasto sanitario ${ }^{3}$.

\section{Objetivos}

Describir el método de determinación de la PVIA y comprobar su utilidad en la vigilancia del acceso vascular.

\section{Material y Métodos}

A un grupo de 31 pacientes portadores de injertos sintéticos se ha realizado de forma programada la medición de la PVIA desde mayo del 2006. En este período hemos contabilizado un total de 31 pacientes con injertos sintéticos

\section{Método de determinación de la PVIA:}

1. Se inicia la diálisis con agujas del calibre 15G, flujo de sangre de $200 \mathrm{ml} / \mathrm{min}$ y se hace la determinación durante los 5 primeros minutos de diálisis, tras haber comprobado la presión venosa del monitor.

2. Con la bomba de sangre parada se pinza la línea venosa entre el dializador y la cámara venosa, se retira la línea del clamp venoso, transcurridos unos 30 segundos se mide la presión del transductor venoso del monitor $(P)$.

3. A continuación, se mide la presión hidrostática (H) que contiene la columna de sangre entre el acceso vascular y la cámara de sangre venosa.

4. Calculamos la PIA equivalente (ePIA) según fórmula adaptada de Besarab $A$ y cols co $^{4}$ :

$$
\mathrm{ePIA}=\mathrm{P}+(0,35 \times \mathbf{H}+3,4)
$$

5. Medimos la presión arterial media (PAM) en $\mathrm{mmHg}$ :

$$
\text { PAM }=[(\text { P. sistólica }+(2 \times \mathbf{P} \text { diastólica })] / 3
$$

6. Para que el valor de la PVIA sea concluyente, por último, calculamos el índice ePIA/PAM.

Si el valor de dicho índice en tres mediciones seguidas es $>0,5$ y/o hay un incremento del $>0,25$ respecto al basal es indicativo del deterioro de la prótesis y hace falta realizar pruebas de imagen.

\section{Resultados}

De los 31 pacientes con injerto estudiados, a 7 se les paró la prótesis por trombosis, no siendo recuperable por angiorradiología. A uno se le realizó un cierre quirúrgico por problemas isquémicos la mano. En estos 7 pacientes, el cálculo de la PVIA daba un resultado superior a 0,5.

El año 2007, se inició con un total de 18 pacientes portadores de prótesis, en el transcurso del año aumentaron hasta 24, se perdieron 8 por trombosis 0 fallecimiento del paciente por lo que finalizó el año con 16 prótesis que representan un $8,9 \%$ del total de los accesos vasculares del centro.

De los 14 pacientes que actualmente llevan prótesis funcionantes, 9 han tenido, en algún momento, una PVIA patológica, haciéndoseles a 4 una angioplastia preventiva, siendo efectiva en todos.

Presentamos a continuación tres situaciones que ilustran el manejo y la información que ha proporcionado la PVIA, en nuestro centro.

\section{Paciente 1}

Portadora de una prótesis Vectra ${ }^{\circledR}$, realizada el 02/11/2004 y puncionada por primera vez el 08/11/2004, actualmente está funcionante. A partir de mayo de 2006 se obtienen valores, entre 0,14 y 0,43 , siendo éstos completamente normales. En marzo del 2007 de forma puntual se detecta una PVIA con un valor de 0,79 , que se atribuyó a problemas de punción o a la presencia de hematomas residuales (figural).

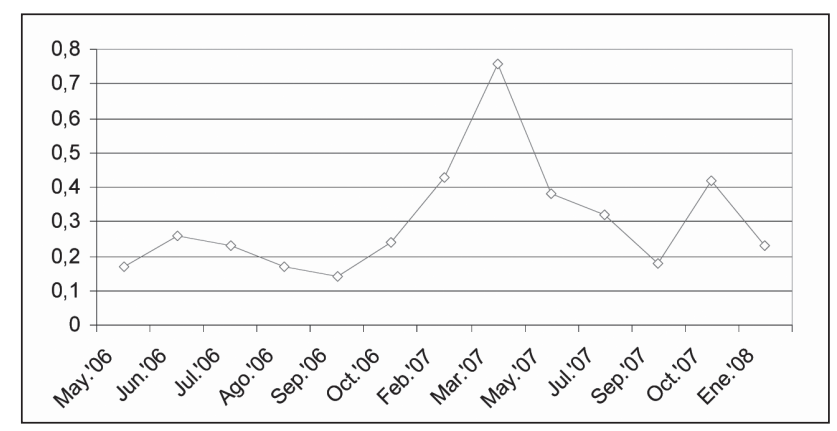

Figura 1. Seguimiento de la PVIA en el paciente 1 


\section{Paciente 2}

Portador de una prótesis tipo Goretex ${ }^{\circledR}$, realizada el 27/03/2007 y puncionada por primera vez el 25/04/2007. Se obtienen valores de PVIA entre 0,19 y 0,29 (figura 2). Tras ocho meses de punciones con valores de PVIA normales, se paró el acceso sin síntomas de trombosis u obstrucción previos. Se produjo otra interrupción del flujo a los 20 días de intervalo, las dos veces se pudo recuperar el acceso por angioplastia y trombólisis en nuestro hospital de referencia. Ante la primera parada, el servicio de angiografía nos informó de la presencia de un hematoma en la zona arterial como posible causa de la trombosis. En el segundo episodio de trombosis se había observado que el día anterior al fallo de la fístula se había tenido que realizar una hemostasia de más de una hora por lo que pensamos que una posible causa de la trombosis pudo ser el exceso de presión sobre la prótesis.

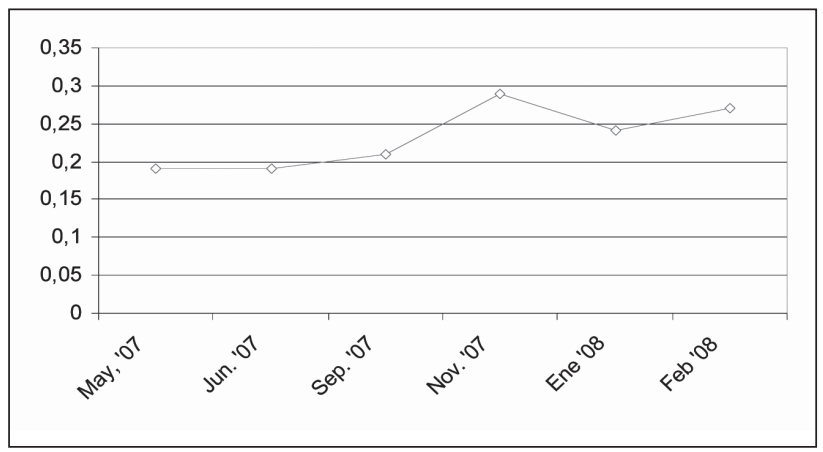

Figura 2. Seguimiento de la PVIA en el paciente 2

\section{Paciente 3}

Portador de una prótesis tipo Goretex ${ }^{\circledR}$, realizado el 23/01/2007 y puncionado por primera vez el 21/02/2007. Se obtienen valores de PVIA entre 0,55 y 0,86, practicándosele una angioplastia en junio del 2007, con un valor posterior límite de 0,5 . Los valores de PVIA continuaron aumentando hasta alcanzar la cifra de 0,88, tras la cual se le practicó una segunda angioplastia en febrero del 2008, siendo efectiva, ya que se normalizaron los valores de PVIA (figura 3). Ante la posibilidad de que el problema radique en la anastomosis venosa y aunque la PVIA sea normal pensamos que este paciente tiene mayor riesgo de sufrir un fallo del acceso vascular.

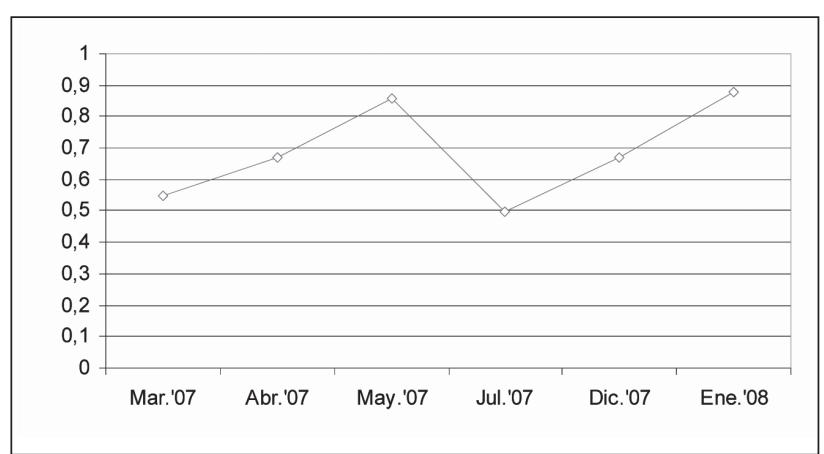

Figura 3. Seguimiento de la PVIA en el paciente 3

\section{Discusión}

La medición de la presión venosa intra-acceso se utiliza principalmente para detectar precozmente la disfunción del injerto sintético con la finalidad de garantizar una adecuada diálisis, y alertar sobre posibles lesiones estructurales tratables, y de esta manera poder prevenir la trombosis y aumentar la supervivencia de éste ${ }^{1,2,6}$.

Aunque en algunos pacientes se detectó a tiempo el problema y se realizó la correspondiente angioplastia, esto no fue garantía para la supervivencia del injerto que acabó fallando.

Como indican otros autores la principal conclusión a la que llegamos es que gracias a la PVIA es posible, en ocasiones, detectar precozmente una disfunción del injerto y actuar debidamente. La determinación de una PVIA elevada y el aumento de la presión venosa, a lo largo de las diferentes sesiones de diálisis, son las características más importantes y determinantes para establecer un problema en la prótesis. Ante la detección de un aumento de la presión venosa del injerto, es necesaria una mayor atención a éste ${ }^{6,7}$. La realización sistemática de la PVIA, nos permitirá comprobar la disfunción y actuar ante ella.

Se ha de tener en cuenta que según el lugar de punción el valor de la PVIA varía, ya que si la punción se realiza en la zona más puncionada o donde se sospecha la estenosis y/o trombosis, ésta será superior. Cabe destacar la importancia de la rotación de las punciones (recomendación que se puede extrapolar a cualquier tipo de acceso), ya que favorece una mayor supervivencia de la prótesis ${ }^{2,6,7}$. Cuando tenemos una zona de punción, con mayor PV o PVIA elevada, es importante intentar evitarla. 


\section{Agradecimientos}

A todas las compañeras, tanto enfermeras como equipo médico y supervisión, que han colaborado en la recogida de datos y participado en este proyecto.

\section{Bibliografía}

1. Armada E et al. Programa de monitorización de accesos vasculares nativos para hemodiálisis. $\mathrm{Ne}-$ frología 2005; XXV (1): 57-66.

2. Rodríguez Hernández JA et al. Guías de acceso vascular en hemodiálisis de la SEN. Nefrología 2005; XXV (Supl.1).

3. Motellón JL et al. Utilidad de la angioplastia transluminal percutánea en el tratamiento de las estenosis de las fístulas arteriovenosas para hemodiálisis. Nefrología 1996; XVI (1): 54-60.
4. Caro $P$ et al. La utilidad de la presión intra-acceso. Nefrología 2004; XXIV (4): 357-363.

5. Górriz JL et al. Ritmo estacional en la trombosis del acceso vascular para hemodiálisis. Nefrología 2007; XXVII (1): 62-67.

6. Gruss E et al. Seguimiento prospectivo del acceso vascular de hemodiálisis mediante un equipo multidisciplinar. Nefrología 2006; XXVI (6): 703-710.

7. Tordoir $\mathrm{J}$ et al. EBPG on vascular access. Rev Nephrology Dialysis Transplantation 2007; 22 (Supl. 2): 88-117. 